Centre de Recherches Mathématiques CRM Proceedings and Lecture Notes

Volume 37, 2004

\title{
The Prolate Spheroidal Phenomenon as a Consequence of Bispectrality
}

\author{
F. Alberto Grünbaum and MiLen Yakimov
}

\begin{abstract}
In this paper we announce that a very large class of integral operators derived from bispectral algebras of rank 1 and 2 (parametrized by Lagrangian Grassmannians of infinitely large size) posses commuting differential operators. The examples of Landau, Pollak, Slepian, and Tracy, Widom, used in time-band limiting and random matrix theory arise as special cases of this result.
\end{abstract}

\section{Introduction}

It was discovered by Landau, Pollak, Slepian, and Tracy, Widom, that certain integral operators associated to the Airy and Bessel special functions possess commuting differential operators. They found important applications of this to time-band limiting, and to the study of asymptotics of Fredholm determinants, relevant to scaling limits of random matrix models. We call this phenomenon the prolate spheroidal phenomenon.

On the other hand, the problem of bispectrality was posed [7] about 20 years ago by one of us (A. G.) and J. J. Duistermaat as a tool to understand this prolate spheroidal property of integral operators. The aim was to extend it to larger classes and search for possible applications. Despite the dramatic recent developments in the areas of random matrices and bispectrality the two problems remained isolated except for several common examples, see [9-11]. In addition only a few integral operators possessing a commuting differential operator were found.

Here we announce that any self-adjoint bispectral algebra of ordinary differential operators (see Definition 3.3 and Definition 4.3 and Section 2 for general definitions) of rank 1 and 2 induces an integral operator possessing the prolate spheroidal property. The kernel of such an operator is of the form

$$
K(x, z)=\int_{\Gamma_{2}} \Psi(x, z) \Psi(y, z) \mathrm{d} z
$$

where $\Psi(x, z)$ is the corresponding bispectral (eigen)function and $\Gamma_{2}$ is a contour in the complex plane with 1 or 2 end points. It acts on the space $L^{2}\left(\Gamma_{1}\right)$ again for

2000 Mathematics Subject Classification. Primary 47G10; Secondary 15A52, 37K10.

This is the final form of the paper. 
a contour with the same property. The main results are stated in Theorem 3.8 and Theorem 4.8.

The integral operators of Landau, Pollak, Slepian, and Tracy, Widom correspond to the cases $\Psi(x, z)=\sqrt{x z} J_{\nu+1 / 2}(i x z)$ and $\Psi(x, z)=A(x+z)$ (in terms of the Bessel functions of first kind and the Airy function) which are known to be "basic" bispectral functions, in the sense that the other rank 1 and 2 bispectral functions are obtained from them by certain types of Darboux transformations (see $[\mathbf{3}, \mathbf{4}, \mathbf{7}, \mathbf{1 4}, \mathbf{2 3}])$. Although in these cases $[\mathbf{1 5}, \mathbf{1 6}, \mathbf{1 9}-\mathbf{2 1}]$ the commuting differential operator is of order 2 , in general it is of arbitrarily large order.

In the rest of the introduction we describe our strategy for proving Theorem 3.8 and Theorem 4.8 which relies on a very interesting property of the "size" of bispectral algebras of rank 1 and 2 .

Consider, more generally, a holomorphic function $\Psi(x, z)$ in some domain of $\mathbb{C} \times \mathbb{C}$ which is not an eigenfunction of any differential operator in $x$ or $z$. Denote by $\mathcal{B}_{\Psi}$ the algebra of differential operators $R\left(x, \partial_{x}\right)$ with rational coefficients for which there exists an operator $S\left(z, \partial_{z}\right)$ with rational coefficients such that

$$
R\left(x, \partial_{x}\right) \Psi(x, z)=S\left(z, \partial_{z}\right) \Psi(x, z) .
$$

The algebra of all differential operators $S\left(z, \partial_{z}\right)$ obtained in this way will be denoted by $\mathcal{C}_{\Psi}$. The equality

$$
b_{\Psi}\left(R\left(x, \partial_{x}\right)\right):=S\left(z, \partial_{z}\right)
$$

correctly defines an anti-isomorphism from $\mathcal{B}_{\Psi}$ to $C_{\Psi}$. Recall that such a function $\Psi(x, z)$ is called bispectral if both algebras $\mathcal{B}_{\Psi}$ and $\mathcal{C}_{\Psi}$ contain rational functions. The subalgebra of $\mathcal{B}_{\Psi}$ and $\mathcal{C}_{\Psi}$, consisting of differential operators in $x$ and $z$ for which $\Psi(x, z)$ is an eigenfunction, are commutative. Algebras obtained in this way are called bispectral algebras. Their rank (which is equal and is also called rank of the bispectral function $\Psi(x, z))$ is the greatest common divisor of the orders of the operators of these algebras.

We derive our main result from the following remarkable property:

Property. Consider the $\mathbb{Z}_{+} \times \mathbb{Z}_{+}$filtration of the algebra $\mathcal{B}_{\Psi}$ given by

$$
\mathcal{B}_{\Psi}^{l_{1}, l_{2}}=\left\{R\left(x, \partial_{x}\right) \in \mathcal{B}_{\Psi} \mid \operatorname{ord} R\left(x, \partial_{x}\right) \leq 2 l_{1}, \operatorname{ord}\left(b_{\Psi} R\right)\left(x, \partial_{x}\right) \leq 2 l_{2}\right\} .
$$

If $\Psi(x, z)$ is a bispectral function of rank $r=1$ or 2 then the the size of the spaces $\mathcal{B}_{\Psi}^{l_{1}, l_{2}}$ in this filtration is large in the sense

$$
\operatorname{dim} \mathcal{B}_{\Psi}^{l_{1}, l_{2}} \geq \frac{2}{r}\left(2 l_{1} l_{2}+l_{1}+l_{2}\right)-\text { const }
$$

where the constant is independent of $l_{1}$ and $l_{2}$.

For the basic bispectral functions $\exp (x z), A(x+z)$, and $\sqrt{x z} J_{\nu+1 / 2}(i x z)$, $\nu \in \mathbb{C} \backslash \mathbb{Z}$ of rank 1 and 2 , the dimension of these spaces is exactly equal to the righthand side with const $=-1$. This is remarkable since it shows that for all bispectral functions $\Psi(x, z)$ of rank 1 and 2 the spaces of the above natural filtration of the algebra $\mathcal{B}_{\Psi}$ are almost of the same dimension as the spaces corresponding to the basic bispectral functions.

It is very interesting to understand the relation between our results and the approach of Adler, Shiota, and van Moerbeke $[\mathbf{1}, \mathbf{2}]$ to the Tracy-Widom system of differential equations via Virasoro constraints. One possible way would be to 
incorporate the representation theoretic meaning of the Bessel tau functions from [5] in terms of representations of the $W_{1+\infty}$ algebra, see [8].

Another important problem is to understand the relation of this work to the isomonodromic deformations approach to random matrices from the works of Palmer [18], Harnad, Tracy, and Widom [13,22], Its and Harnad [12], and Borodin and Deift $[6]$.

The proofs of the results announced here will appear in a forthcoming publication.

Acknowledgments. M. Y. would like to thank the organizers of the Workshop on Superintegrability, September 2002, CRM, Montreal and especially John Harnad and Pavel Winternitz for the opportunity to participate at the conference and for their interest in this work.

\section{Bispectrality and commutativity}

Denote by $\mathcal{W}_{\text {rat }}$ the algebra of differential operators in one variable with rational coefficients. Denote by $a(\cdot)$ the formal adjoint of operators in $\mathcal{W}_{\text {rat }}$ :

$$
a\left(\sum_{k=0}^{n} b_{k}(x) \partial_{x}^{n}\right)=\sum_{k=0}^{n}\left(-\partial_{x}\right)^{n} b_{k}(x) .
$$

For any $\xi \in \mathbb{C}$ consider the map from the subalgebra of $\mathcal{W}_{\text {rat }}$ consisting of operators regular at $\xi$ to the set of bidifferential operators at $\xi$

$$
\phi_{\xi}\left(\sum_{k=0}^{n} b_{k}(x) \partial_{x}^{n}\right)=\left.\sum_{k=0}^{n} \sum_{i=0}^{k-1}(-1)^{i} \partial_{x}^{k-i-1} \otimes \partial_{x}^{i} b_{k}(x)\right|_{x=\xi} .
$$

For an oriented contour $\Gamma$ in $\mathbb{C}$ we will denote its endpoints by $e(\Gamma)$. For any $\xi \in e(\Gamma)$ set $\pi(\xi)=1$ or 0 depending on whether $\xi$ is a left or right endpoint of $\Gamma$.

Assume that

$$
D\left(x, \partial_{x}\right)=\sum_{k=0}^{n} b_{k}(x) \partial_{x}^{n} \in \mathcal{W}_{\text {rat }}
$$

is a differential operator which is regular along $\Gamma$. Let $f(x)$ and $g(x)$ be smooth functions on $\Gamma$ that decrease rapidly when $x \rightarrow \infty$ (i.e., $\lim _{x \rightarrow \infty} p(x) f^{(k)}(x)=0$ for any polynomial $p(x)$ and any integer $k$, similarly for $g(x))$.

By standard integration by parts one gets that

$$
\begin{aligned}
\int_{\Gamma}\left(D\left(x, \partial_{x}\right) f(x)\right) g(x) \mathrm{d} x=\sum_{\xi \in e(\Gamma)}(-1)^{\pi(\xi)} \phi_{\xi}\left(D\left(x, \partial_{x}\right)\right)(f(x) \otimes g(x)) \\
+\int_{\Gamma} f(x)\left(a D\left(x, \partial_{x}\right) g(x)\right) \mathrm{d} x .
\end{aligned}
$$

Let $\Psi(x, z)$ be a holomorphic function in some domain of $\mathbb{C} \times \mathbb{C}$ which is not an eigenfunction of a differential operator in $x$ or $z$, as in the introduction. Recall the definition (1.1) of the algebras of differential operators $\mathcal{B}_{\Psi}$ and $\mathcal{C}_{\Psi}$. Assume that $\widetilde{\mathcal{B}}_{\Psi}$ and $\widetilde{\mathcal{C}}_{\Psi}$ are two subalgebras of $\mathcal{B}_{\Psi}$ and $\mathcal{C}_{\Psi}$, respectively, that are stable under the formal adjoint map (2.1) and such that

$$
b_{\Psi}\left(\widetilde{\mathcal{B}}_{\Psi}\right)=\widetilde{\mathcal{C}}_{\Psi} .
$$


Proposition 2.1. Let $\Gamma_{1}$ and $\Gamma_{2}$ be two contours in $\mathbb{C}$ such that $\Gamma_{1} \times \Gamma_{2}$ is in the domain of $\Psi(x, z)$ and $\Psi(x, z)$ decreases rapidly when $x$ or $z$ go to $\infty$ along $\Gamma_{1}$ and $\Gamma_{2}$. If $D\left(x, \partial_{x}\right) \in \widetilde{\mathcal{B}}_{\Psi}$ is such that

$$
a b_{\Psi}\left(D\left(x, \partial_{x}\right)\right)=b_{\Psi} a\left(D\left(x, \partial_{x}\right)\right)
$$

$$
\phi_{\xi}\left(D\left(x, \partial_{x}\right)\right)=0, \forall \xi \in e\left(\Gamma_{1}\right) ; \quad \phi_{\xi}\left((b D)\left(z, \partial_{z}\right)\right)=0, \forall \xi \in e\left(\Gamma_{2}\right)
$$

then the integral operator with kernel

$$
K(x, y)=\int_{\Gamma_{2}} \Psi(x, z) \Psi(y, z) \mathrm{d} z
$$

on $L^{2}\left(\Gamma_{1}\right)$ commutes with the differential operator $D\left(x, \partial_{x}\right)$ with domain all smooth functions on $\Gamma_{1}$ that decrease rapidly as $x \rightarrow \infty$.

Let us call a differential operator $D\left(x, \partial_{x}\right) \in \mathcal{W}_{\text {rat }}$ formally symmetric if

$$
(a D)\left(x, \partial_{x}\right)=D\left(x, \partial_{x}\right)
$$

and formally skewsymmetric if

$$
(a D)\left(x, \partial_{x}\right)=-D\left(x, \partial_{x}\right) .
$$

Lemma 2.2. $\quad$ (i) A differential operator $D\left(x, \partial_{x}\right) \in \mathcal{W}_{\text {rat }}$ is formally symmetric if and only if it has the form

$$
D\left(x, \partial_{x}\right)=\sum_{i=0}^{n} \partial_{x}^{n} c_{i}(x) \partial_{x}^{n}
$$

for some integer $n$ and some rational functions $c_{i}(x)$.

(ii) The operator $D\left(x, \partial_{x}\right)$ given by (2.7) satisfies

$$
\phi_{\xi}\left(D\left(x, \partial_{x}\right)\right)=0
$$

for some fixed $\xi \in \mathbb{C}$ if and only if

$$
\left(\partial_{x}^{i} c_{k}\right)(\xi)=0 \quad \text { for } k=1, \ldots, n, i=0, \ldots, k-1 .
$$

For a given function $\Psi(x, z)$ as before let $\mathcal{B}_{\Psi \text {,sym }}$ be the subalgebra of $\mathcal{B}_{\Psi}$ consisting of differential operators $R\left(x, \partial_{x}\right)$ for which both $R\left(x, \partial_{x}\right)$ and $\left(b_{\Psi} R\right)\left(z, \partial_{z}\right)$ are formally symmetric. Set also $\mathcal{C}_{\Psi, \mathrm{sym}}:=b_{\Psi}\left(\mathcal{B}_{\Psi, \mathrm{sym}}\right)$. Define the vector spaces

$$
\begin{gathered}
\mathcal{B}_{\Psi, \mathrm{sym}}^{l_{1}, l_{2}}=\left\{R\left(x, \partial_{x}\right) \in \mathcal{B}_{\Psi, \mathrm{sym}} \mid \operatorname{ord} R\left(x, \partial_{x}\right) \leq 2 l_{1}\right. \\
\text { and } \left.\operatorname{ord}\left(b_{\Psi} R\right)\left(z, \partial_{z}\right) \leq 2 l_{2}\right\}, \\
\mathcal{C}_{\Psi, \mathrm{sym}}^{l_{1}, l_{2}}=\left\{S\left(z, \partial_{z}\right) \in \mathcal{C}_{\Psi, \mathrm{sym}} \mid \operatorname{ord}\left(b_{\Psi}^{-1} S\right)\left(x, \partial_{x}\right) \leq 2 l_{1}\right. \\
\text { and } \left.\operatorname{ord} S\left(z, \partial_{z}\right) \leq 2 l_{2}\right\} .
\end{gathered}
$$

Clearly $\mathcal{C}_{\Psi, \text { sym }}^{l_{1}, l_{2}}=b_{\Psi}\left(\mathcal{B}_{\Psi, \text { sym }}^{l_{1}, l_{2}}\right)$.

¿From Proposition 2.1 and Lemma 2.2 we obtain:

THEOREM 2.3. Assume the conditions from Proposition 2.1 for the the function $\Psi(x, z)$ and the contours $\Gamma_{1}$ and $\Gamma_{2}$. If either of the following two conditions is satisfied then the integral operator with kernel (2.6) possesses a formally commuting symmetric differential operator of order less than or equal to $2 l_{1}$ and domain-the space of rapidly decreasing smooth functions on $\Gamma_{1}$ 
THE PROLATE SPHEROIDAL PHENOMENON AS A CONSEQUENCE OF BISPECTRALIT3Ø7

Condition (i)

$$
\mathcal{B}_{\Psi, \mathrm{sym}}^{l_{1}, l_{2}}>l_{1}\left(l_{1}+1\right) e\left(\Gamma_{1}\right) / 2+l_{2}\left(l_{2}+1\right) e\left(\Gamma_{2}\right) / 2+1
$$

Condition (ii) $-e\left(\Gamma_{1}\right)=e\left(\Gamma_{1}\right),-e\left(\Gamma_{2}\right)=e\left(\Gamma_{2}\right)$, all operators in $\mathcal{B}_{\Psi, \text { sym }}$ are invariant under the transformation $x \mapsto-x$, and

$$
\mathcal{B}_{\Psi, \mathrm{sym}}^{l_{1}, l_{2}}>l_{1}\left(l_{1}+1\right) e\left(\Gamma_{1}\right) / 4+l_{2}\left(l_{2}+1\right) e\left(\Gamma_{2}\right) / 4+1 \text {. }
$$

\section{Integral operators associated to self-adjoint Darboux transformations of Airy functions}

3.1. The Airy bispectral function. Denote by $A(x)$ the Airy function and set

$$
\Psi_{A}(x, z)=A(x+z) .
$$

Recall that $A(x)$ decreases rapidly when $x \rightarrow \infty$ in the sector $-\pi / 3<\arg x<\pi / 3$.

If $L_{A}\left(x, \partial_{x}\right)$ denotes the Airy differential operator

$$
L_{A}\left(x, \partial_{x}\right)=\partial_{x}^{2}-x
$$

then $\Psi_{A}(x, z)$ satisfies

$$
\begin{gathered}
L_{A}\left(x, \partial_{x}\right) \Psi_{A}(x, z)=z \Psi_{A}(x, z), \\
\partial_{x} \Psi_{A}(x, z)=\partial_{z} \Psi_{A}(x, z), \\
x \Psi_{A}(x, z)=L_{A}\left(z, \partial_{z}\right) \Psi_{A}(x, z) .
\end{gathered}
$$

For shortness denote the algebras $\mathcal{B}_{\Psi_{A}}$ and $\mathcal{C}_{\Psi_{A}}$ of differential operators with rational coefficients associated to the Airy function $\Psi_{A}(x, z)$, recall $(1.1)$, by $\mathcal{B}_{A}$ and $\mathcal{C}_{A}$. It is straightforward to deduce:

LEMMA 3.1. The algebras $\mathcal{B}_{A}$ and $\mathcal{C}_{A}$ coincide with the Weyl algebra $\mathcal{W}_{\text {poly }}$ of differential operators in one variable with polynomial coefficients. Moreover the anti-isomorphism $b_{A}$ associated to the Airy function $\Psi_{A}(x, z)$, recall (3.1), is uniquely defined from the relations

$$
b_{A}(x)=\left(L_{A}\left(z, \partial_{z}\right)\right), \quad b_{A}\left(\partial_{x}\right)=\partial_{z}, \quad b_{A}\left(L_{A}\left(x, \partial_{x}\right)\right)=z .
$$

3.2. Self-adjoint Darboux transformations from the Airy function. Note that

$$
\mathbb{C}[x]=\mathcal{B}_{A} \cap \mathbb{C}(x)
$$

and

$$
\mathbb{C}\left[L_{A}\left(x, \partial_{x}\right)\right]=b_{A}^{-1}\left(\mathcal{C}_{A} \cap \mathbb{C}(z)\right) .
$$

The set of rational Darboux transformations $\mathcal{D}_{A}$ from the Airy function was defined in $[4]$ as the set of functions $\Psi(x, z)$ for which there exist differential operators

$$
P\left(x, \partial_{x}\right), Q\left(x, \partial_{x}\right) \in(\mathbb{C}[x] \backslash 0)^{-1} \mathcal{B}_{A}=\mathcal{W}_{\text {rat }}
$$

such that

$$
\begin{aligned}
& f\left(L_{A}\left(x, \partial_{x}\right)\right)=Q\left(x, \partial_{x}\right) P\left(x, \partial_{x}\right), \\
& \Psi(x, z)=\frac{1}{p(z)} P\left(x, \partial_{x}\right) \Psi_{A}(x, z),
\end{aligned}
$$


for some polynomials $f(t)$ and $p(z)$. (The polynomial $p(z)$ is included for normalization purposes only.) The quotient ring of $\mathcal{B}_{A}$ by $\mathbb{C}[x] \backslash\{0\}$ in (3.2) is well defined since $\mathbb{C}[x] \backslash\{0\}$ is an Ore subset of $\mathcal{B}_{A}$, see $[\mathbf{1 7}]$.

It was also shown in $[\mathbf{4}, \mathbf{1 4}]$ and more conceptually proved in $[\mathbf{3}]$ that:

THEOREM 3.2. All rational Darboux transformations from the Airy function $\Psi(x, z)$ are bispectral functions of rank 2 .

Definition 3.3. Define the set $\mathcal{S D}_{A}$ of self-adjoint Darboux transformations from the Airy function $\Psi(x, z)$ to consist of those functions $\Psi(x, z)$ for which there exists a differential operator $P\left(x, \partial_{x}\right) \in \mathcal{W}_{\text {rat }}$ such that

$$
\begin{gathered}
g\left(L_{A}\left(x, \partial_{x}\right)\right)^{2}=(a P)\left(x, \partial_{x}\right) P\left(x, \partial_{x}\right), \\
\Psi(x, z)=\frac{1}{g(z)} P\left(x, \partial_{x}\right) \Psi_{A}(x, z),
\end{gathered}
$$

for some polynomial $g(t)$.

In fact $\mathcal{S D}_{A}$ consists exactly of those $\Psi(x, z) \in \mathcal{D}_{A}$ for which $Q\left(x, \partial_{x}\right)=$ $(a P)\left(x, \partial_{x}\right)$ in $(3.3)-(3.4)$ with an appropriate normalization of the polynomial $p(z)$. One can show that as a consequence $f(t)$ is the square of some polynomial $g(t)$, compare to $(3.5)-(3.6)$.

3.3. Size of the algebra $\mathcal{B}_{A}$ relative to the anti-isomorphism $\boldsymbol{b}_{A}$. Consider the $\mathbb{Z}_{+} \times \mathbb{Z}_{+}$filtration of the algebra $\mathcal{B}_{A}$ associated to the Airy function $\Psi_{A}(x, z)$, defined analogously to $(2.8)$ by

$$
\mathcal{B}_{A}^{l_{1}, l_{2}}=\left\{R\left(x, \partial_{x}\right) \in \mathcal{B}_{A} \mid \operatorname{ord} R\left(x, \partial_{x}\right) \leq 2 l_{1}, \operatorname{ord}\left(b_{A} R\right)\left(z, \partial_{z}\right) \leq 2 l_{2}\right\} .
$$

LEMMA 3.4. The vector space $\mathcal{B}_{A}^{l_{1}, l_{2}}$ has a basis that consists of the differential operators

$$
\left\{x^{m}\left(L_{A}\left(x, \partial_{x}\right)\right)^{n} \mid n \leq l_{1}, m \leq l_{2}\right\} \cup\left\{x^{m} \partial_{x}\left(L_{A}\left(x, \partial_{x}\right)\right)^{n} \mid n<l_{1}, m<l_{2}\right\} .
$$

Note that the formal adjoint anti-involution $a$ of $\mathcal{W}_{\text {rat }}$ preserves the spaces $\mathcal{B}_{A}^{l_{1}, l_{2}}$. Since $a^{2}=$ id the space $\mathcal{B}_{A}^{l_{1}, l_{2}}$ is the direct sum of the eigenspaces of $a$ with eigenvalues \pm 1 . Denote the eigenvalue 1 subspace of $\mathcal{B}_{A}^{l_{1}, l_{2}}$ by $\mathcal{B}_{A, \mathrm{sym}}^{l_{1}, l_{2}}$. For the Airy function $\Psi_{A}(x, z)$ one has $a b_{A}=b_{A} a$ and thus:

$$
\begin{aligned}
\mathcal{B}_{A, \mathrm{sym}}^{l_{1}, l_{2}}=\left\{R\left(x, \partial_{x}\right) \in \mathcal{B}_{A} \mid \operatorname{ord} R\left(x, \partial_{x}\right) \leq 2 l_{1}, \operatorname{ord}\left(b_{A} R\right)\left(z, \partial_{z}\right) \leq 2 l_{2},\right. \\
\left.a R\left(x, \partial_{x}\right)=R\left(x, \partial_{x}\right), a\left(b_{A} R\left(z, \partial_{z}\right)\right)=b_{A} R\left(z, \partial_{z}\right)\right\} .
\end{aligned}
$$

LEMMA 3.5. The set of operators

$$
\left\{x^{m}\left(L_{A}\left(x, \partial_{x}\right)\right)^{n}+\left(L_{A}\left(x, \partial_{x}\right)\right)^{n} x^{m} \mid n \leq l_{1}, m \leq l_{2}\right\}
$$

is a basis for the space $\mathcal{B}_{A, \mathrm{sym}}^{l_{1}, l_{2}}$. In particular

$$
\operatorname{dim} \mathcal{B}_{A, \mathrm{sym}}^{l_{1}, l_{2}}=\left(l_{1}+1\right)\left(l_{2}+1\right) .
$$


THE PROLATE SPHEROIDAL PHENOMENON AS A CONSEQUENCE OF BISPECTRALITßø9

3.4. Size of the algebra $\mathcal{B}_{\Psi, \text { sym }}$ for a self-adjoint Darboux transformation from the Airy function, relative to the involution $b_{\Psi}$. Fix an arbitrary self-adjoint Darboux transformation from the Airy function $\Psi \in \mathcal{S D}_{A}$ given by (3.5)-(3.6) for some $P\left(x, \partial_{x}\right) \in \mathcal{W}_{\text {rat }}$ and $g(t) \in \mathbb{C}[t]$. Let

$$
P\left(x, \partial_{x}\right)=\frac{1}{v(x)} R\left(x, \partial_{x}\right)
$$

for some $R\left(x, \partial_{x}\right) \in \mathcal{W}_{\text {poly }}=\mathcal{B}_{A}$ and $v(x) \in \mathbb{C}[x]$. Set

$$
\operatorname{ord} R\left(x, \partial_{x}\right)=\rho_{1} \quad \text { and } \quad \operatorname{ord}\left(b_{A} R\right)\left(x, \partial_{x}\right)=\rho_{2} .
$$

Denote

$$
\begin{aligned}
& \mathcal{S}_{\Psi, 1}=\operatorname{Span}\left\{\frac{1}{v(x)} R\left(x, \partial_{x}\right) M\left(x, \partial_{x}\right)(a R)\left(x, \partial_{x}\right) \frac{1}{v(x)} \mid M\left(x, \partial_{x}\right) \in \mathcal{B}_{A, \mathrm{sym}}^{l_{1}-\rho_{1}, l_{2}}\right\}, \\
& \mathcal{S}_{\Psi, 2}=\operatorname{Span}\left\{v(x) M\left(x, \partial_{x}\right) v(x) \mid M\left(x, \partial_{x}\right) \in \mathcal{B}_{A, \mathrm{sym}}^{l_{1}, l_{2}-\rho_{2}}\right\} .
\end{aligned}
$$

Proposition 3.6. In the above setting:

(i) The spaces of differential operators $\mathcal{S}_{\Psi, 1}$ and $\mathcal{S}_{\Psi, 2}$ are subspaces of $\mathcal{B}_{\Psi, \mathrm{sym}}^{l_{1}, l_{2}}$.

(ii) The dimension of the intersection $\mathcal{S}_{\Psi, 1} \cap \mathcal{S}_{\Psi, 2}$ is less than or equal to

$$
\left(l_{1}-\rho_{1}+1\right)\left(l_{2}-\rho_{2}+1\right) \text {. }
$$

Theorem 4.2 of $[\mathbf{3}]$ shows that (3.5)-(3.6) is equivalent to

$$
\begin{gathered}
v\left(L_{A}\left(z, \partial_{z}\right)\right)^{2}=\left(a b_{A} R\right)\left(z, \partial_{z}\right) \frac{1}{g(z)^{2}}\left(b_{A} R\right)\left(z, \partial_{z}\right), \\
\Psi(x, z)=\frac{1}{v(x) g(z)}\left(b_{A} R\right)\left(z, \partial_{z}\right) \Psi_{A}(x, z) .
\end{gathered}
$$

(The hard step is to prove the first equality.) One can show that

$$
b_{\Psi}\left(\frac{1}{v(x)} R\left(x, \partial_{x}\right) M\left(x, \partial_{x}\right)(a R)\left(x, \partial_{x}\right) \frac{1}{v(x)}\right)=g(z)\left(b_{A} M\right)\left(z, \partial_{z}\right) g(z)
$$

for all operators $M\left(x, \partial_{x}\right) \in \mathcal{B}_{A}$. The fact that $\mathcal{S}_{\Psi, 2}$ is a subspace of $\mathcal{B}_{\Psi, \text { sym }}^{l_{1}, l_{2}}$ follows from this and the fact that $\mathcal{S}_{\Psi, 1} \subset \mathcal{B}_{\Psi, \text { sym }}^{l_{1}, l_{2}}$ by exchanging the roles of $x$ and $z$.

TheOREm 3.7. For any self-adjoint Darboux transformation from the Airy function $\Psi(x, z) \in \mathcal{S D}_{A}$ the dimension of the space of differential operators $\mathcal{S}_{\Psi, 1}+$ $\mathcal{S}_{\Psi, 2}$ is greater than or equal to $\left(l_{1}+1\right)\left(l_{2}+1\right)-\rho_{1} \rho_{2}$. In particular

$$
\operatorname{dim} \mathcal{B}_{\Psi, \text { sym }}^{l_{1}, l_{2}} \geq\left(l_{1}+1\right)\left(l_{2}+1\right)-\rho_{1} \rho_{2} .
$$

Theorem 3.7 and Theorem 2.3 imply our final result for integral operators derived from Darboux transformations from the Airy function.

TheOREM 3.8. Let $\Psi(x, z) \in \mathcal{S D}_{A}$ be a self-adjoint Darboux transformation from the Airy function, given by (3.5), (3.6), (3.9). Let $\Gamma_{1}, \Gamma_{2}$ be two connected contours in $\mathbb{C}$ that do not contain the roots of the polynomials $v(t)$ and $g(t) r e-$ spectively and that begin at some finite points and go to infinity in the sector $-\pi / 3<\arg x<\pi / 3$. Then the integral operator

$$
K(x, y)=\int_{\Gamma_{2}} \Psi(x, z) \Psi(y, z) \mathrm{d} z
$$


on $L^{2}\left(\Gamma_{1}\right)$ commutes with a formally symmetric differential operator with rational coefficients of order less than or equal to $2\left(\rho_{1} \rho_{2}+1\right)$ and domain all smooth functions on $\Gamma_{1}$ that decrease rapidly as $x \rightarrow \infty$.

\section{Integral operators associated to self-adjoint Darboux transformations of Bessel functions}

4.1. The Bessel bispectral function. Denote by $J_{\nu}$ the standard Bessel functions of first kind. By abuse of notation the functions

$$
\Psi_{\nu}(x, z)=(x z)^{1 / 2} J_{\nu+1 / 2}(i x z)
$$

will be also called Bessel functions. Consider the Euler operator

$$
D_{x}=x \partial_{x}
$$

and the operators

$$
L_{\nu}\left(x, \partial_{x}\right)=\partial_{x}^{2}-\frac{\nu(\nu+1)}{x^{2}}=\frac{1}{x^{2}}\left(D_{x}+\nu\right)\left(D_{x}-\nu-1\right)
$$

to be called Bessel operators. The Bessel functions satisfy the equations

$$
\begin{gathered}
L_{\nu}\left(x, \partial_{x}\right) \Psi_{\nu}(x, z)=z^{2} \Psi_{\nu}(x, z), \\
D_{x} \Psi_{\nu}(x, z)=D_{z} \Psi_{\nu}(x, z), \\
x^{2} \Psi_{\nu}(x, z)=L_{\nu}\left(z, \partial_{z}\right) \Psi_{\nu}(x, z) .
\end{gathered}
$$

For shortness the algebras $\mathcal{B}_{\Psi_{\nu}}$ and $\mathcal{C}_{\Psi_{\nu}}$, associated to the Bessel function $\Psi_{\nu}(x, z)$, recall (1.1), will be denoted by $\mathcal{B}_{\nu}$ and $\mathcal{C}_{\nu}$.

The Bessel functions corresponding to $\nu_{1}, \nu_{2} \in \mathbb{C}$ that differ by an integer can be obtained by a Darboux transformation from each other:

$$
\Psi_{\nu+1}(x, z)=\frac{1}{x z}\left(D_{x}-\nu-1\right) \Psi_{\nu}(x, z), \quad \Psi_{\nu}(x, z)=\frac{1}{x z}\left(D_{x}+\nu+1\right) \Psi_{\nu+1}(x, z),
$$

which corresponds to the factorizations

$$
L_{\nu}=x^{-1}\left(D_{x}+\nu+1\right) x^{-1}\left(D_{x}-\nu-1\right), \quad L_{\nu+1}=x\left(D_{x}-\nu-1\right) x^{-1}\left(D_{x}+\nu+1\right) .
$$

According to (4.3)-(4.5) the algebras of differential operators $\mathcal{B}_{\nu}$ and $\mathcal{C}_{\nu}$ contain the operators $L_{\nu}\left(x, \partial_{x}\right), D_{x}$, and $x^{2}$. Denote their subalgebras generated by those operators by $\widetilde{\mathcal{B}}_{\nu}$ and $\widetilde{\mathcal{C}}_{\nu}$, respectively. Clearly (4.3)-(4.5) imply

$$
b_{\nu}\left(\widetilde{\mathcal{B}}_{\nu}\right)=\widetilde{\mathcal{C}}_{\nu}
$$

Similarly to Proposition 2.4 in [4] one shows:

LEMMA 4.1. (i) For $\nu \in \mathbb{C} \backslash \mathbb{Z}$ the algebras $\mathcal{B}_{\nu}$ and $\mathcal{C}_{\nu}$ are generated by the operators $L_{\nu}\left(x, \partial_{x}\right), D_{x}$, and $x^{2}$, i.e.,

$$
\mathcal{B}_{\nu}=\widetilde{\mathcal{B}}_{\nu}, \quad \mathcal{C}_{\nu}=\widetilde{\mathcal{C}}_{\nu}
$$

(ii) For $\nu \in \mathbb{Z}$ the subalgebras $\widetilde{\mathcal{B}}_{\nu}$ and $\widetilde{\mathcal{C}}_{\nu}$ of $\mathcal{B}_{\nu}$ and $\mathcal{C}_{\nu}$ consist of exactly those differential operators in $\mathcal{B}_{\nu}$ and $\mathcal{C}_{\nu}$ that are invariant under the transformation $x \mapsto-x$.

Note that

$$
a b_{\nu}\left(P\left(x, \partial_{x}\right)\right)=b_{\nu} a\left(P\left(x, \partial_{x}\right)\right), \quad \forall P\left(x, \partial_{x}\right) \in \widetilde{\mathcal{B}}_{\nu}
$$


THE PROLATE SPHEROIDAL PHENOMENON AS A CONSEQUENCE OF BISPECTRALITM1

4.2. Self-adjoint Darboux transformations from the Bessel functions. Similarly to the Airy case we have

$$
\mathbb{C}\left[x^{2}\right]=\widetilde{\mathcal{B}}_{\nu} \cap \mathbb{C}(x), \quad \mathbb{C}\left[L_{\nu}\left(x, \partial_{x}\right)\right]=b_{\nu}^{-1}\left(\widetilde{\mathcal{C}}_{\nu} \cap \mathbb{C}(z)\right) .
$$

The set of rational Darboux transformations $\mathcal{D}_{\nu}$ from the Bessel function $\Psi_{\nu}(x, z)$ is defined to be the set of functions $\Psi(x, z)$ for which there exist differential operators

$$
P\left(x, \partial_{x}\right), Q\left(x, \partial_{x}\right) \in\left(\mathbb{C}\left[x^{2}\right] \backslash\{0\}\right)^{-1} \mathcal{B}_{A}
$$

such that

$$
\begin{aligned}
& f\left(L_{\nu}\left(x, \partial_{x}\right)\right)=Q\left(x, \partial_{x}\right) P\left(x, \partial_{x}\right), \\
& \Psi(x, z)=\frac{1}{p(z)} P\left(x, \partial_{x}\right) \Psi_{\nu}(x, z),
\end{aligned}
$$

for some polynomials $f(t)$ and $p(z)$. Again $\left(\mathbb{C}\left[x^{2}\right] \backslash\{0\}\right)$ is an Ore subset of $\mathcal{B}_{A}$ and quotient ring in (4.7) makes sense.

The following theorem was proved in $[\mathbf{2 3}]$ for $\nu=1$ and in $[\mathbf{3}, \mathbf{4}]$ in the general case.

THEOREM 4.2. All rational Darboux transformations from the Bessel functions are bispectral of rank 2 if $\nu \in \mathbb{C} \backslash \mathbb{Z}$ and of rank 1 if $\nu \in \mathbb{Z}$.

Definition 4.3. Define the set of self-adjoint ("even, self-adjoint" in the case $\nu \in \mathbb{Z})$ Darboux transformations $\mathcal{S D}_{\nu}$ from the Bessel functions $\Psi_{\nu}(x, z)$ to consist of all functions $\Psi(x, z)$ for which there exists a differential operator

$$
P\left(x, \partial_{x}\right) \in\left(\mathbb{C}\left[x^{2}\right] \backslash\{0\}\right)^{-1}\left(\widetilde{B}_{\nu}\right)
$$

such that

$$
\begin{array}{r}
f\left(L_{\nu}\left(x, \partial_{x}\right)\right)=(-1)^{m}(a P)\left(x, \partial_{x}\right) P\left(x, \partial_{x}\right) \\
\Psi(x, z)=\frac{1}{z^{m} g\left(z^{2}\right)} P\left(x, \partial_{x}\right) \Psi_{\nu}(x, z),
\end{array}
$$

for a polynomial $f(t)$ of the form

$$
f(t)=t^{2 m} g\left(t^{2}\right)^{2}, \quad g(0) \neq 0 ; g(t) \in \mathbb{C}[t] .
$$

Here the term "even" reflects the fact that for $\nu \in \mathbb{Z}$ the algebras $\mathcal{B}_{\nu}$ and $\mathcal{C}_{\nu}$ are bigger than $\widetilde{\mathcal{B}}_{\nu}$ and $\widetilde{\mathcal{C}}_{\nu}$. The reason for this terminology is explained below.

As in the Airy case the set $\mathcal{S D}_{\nu}$ consists of those rational Darboux transformations $\Psi(x, z)$ from the Bessel function $\Psi_{\nu}(x, z)$ for which in the notation (4.8)-(4.9)

$$
Q\left(x, \partial_{x}\right)=(-1)^{\text {ord } P}(a P)\left(x, \partial_{x}\right)
$$

with the additional property in the case $\nu \in \mathbb{Z}$

$$
P\left(-x,-\partial_{x}\right)=P\left(x, \partial_{x}\right) .
$$

As a consequence it is obtained that the polynomial $f(t)$ in (4.8) has the form (4.12) and an appropriate normalization of $p(z)$ is made.

Note that Lemma 4.1 implies that in the rank 2 case $\nu \in \mathbb{C} \backslash \mathbb{Z}$ the condition (4.14) is a consequence of (4.7). In the rank 1 case $\nu \in \mathbb{Z}$ the term "even" reflects this extra condition. It is needed since in the case of Darboux transformations from the Bessel function the prolate spheroidal property will be deduced from the second condition in Theorem 2.3. 
4.3. Size of the algebras $\widetilde{B}_{\nu}$ relative to the anti-isomorphisms $b_{\nu}$. Consider the $\mathbb{Z}_{+} \times \mathbb{Z}_{+}$filtration of the algebras $\widetilde{B}_{\nu}$

$$
\widetilde{\mathcal{B}}_{\nu}^{l_{1}, l_{2}}=\left\{R\left(x, \partial_{x}\right) \in \widetilde{\mathcal{B}}_{\nu} \mid \text { ord } R\left(x, \partial_{x}\right) \leq 2 l_{1}, \operatorname{ord}\left(b_{\nu} R\right)\left(z, \partial_{z}\right) \leq 2 l_{2}\right\}
$$

The formal adjoint involution $a$ of $\mathcal{W}_{\text {rat }}$ preserves the spaces $\mathcal{B}_{\nu}^{l_{1}, l_{2}}$. Similarly to Lemma 3.4 one shows:

LEMMA 4.4. The vector space $\mathcal{B}_{\nu}^{l_{1}, l_{2}}$ has a basis that consists of the differential operators

$$
\left\{x^{2 m}\left(L_{\nu}\left(x, \partial_{x}\right)\right)^{n} \mid n \leq l_{1}, m \leq l_{2}\right\} \cup\left\{x^{2 m} D_{x}\left(L_{\nu}\left(x, \partial_{x}\right)\right)^{n} \mid n<l_{1}, m<l_{2}\right\} .
$$

Since $a^{2}=$ id the space $\widetilde{\mathcal{B}}_{\nu}^{l_{1}, l_{2}}$ is the direct sum of the eigenspaces of $a$ with eigenvalues \pm 1 . The eigenvalue 1 subspace of $\widetilde{\mathcal{B}}_{\nu}^{l_{1}, l_{2}}$ will be denoted by $\widetilde{\mathcal{B}}_{\nu, \mathrm{sym}}^{l_{1}, l_{2}}$. The commutativity (4.6) of $a$ and $b_{\nu}$ on $\widetilde{\mathcal{B}}_{\nu}^{l_{1}, l_{2}}$ implies:

$$
\begin{gathered}
\widetilde{\mathcal{B}}_{\nu, \mathrm{sym}}^{l_{1}, l_{2}}=\left\{R\left(x, \partial_{x}\right) \in \widetilde{\mathcal{B}}_{\nu} \mid \operatorname{ord} R\left(x, \partial_{x}\right) \leq 2 l_{1}, \operatorname{ord}\left(b_{\nu} R\right)\left(z, \partial_{z}\right) \leq 2 l_{2},\right. \\
\left.a R\left(x, \partial_{x}\right)=R\left(x, \partial_{x}\right), a\left(b_{\nu} R\left(x, \partial_{x}\right)\right)=b_{\nu} R\left(x, \partial_{x}\right)\right\} .
\end{gathered}
$$

LEMma 4.5. The set of operators

$$
\left\{x^{2 m}\left(L_{\nu}\left(x, \partial_{x}\right)\right)^{n}+\left(L_{\nu}\left(x, \partial_{x}\right)\right)^{n} x^{2 m} \mid n \leq l_{1}, m \leq l_{2}\right\}
$$

is a basis for the space $\widetilde{\mathcal{B}}_{\nu, \mathrm{sym}}^{l_{1}, l_{2}}$. In particular

$$
\operatorname{dim} \widetilde{\mathcal{B}}_{\nu, \mathrm{sym}}^{l_{1}, l_{2}}=\left(l_{1}+1\right)\left(l_{2}+1\right) .
$$

4.4. Size of the algebra $\mathcal{B}_{\Psi, \mathrm{sym}}$ for an (even) self-adjoint Darboux transformation from a Bessel function. Fix an arbitrary self-adjoint (and in addition even in the rank 1 case $\nu \in \mathbb{Z}$ ) Darboux transformation from a Bessel function $\Psi_{\nu}(x, z), \Psi \in \mathcal{S D}_{\nu}$ given by (4.10)-(4.11) for some $P\left(x, \partial_{x}\right) \in\left(\mathbb{C}\left[x^{2}\right] \backslash\{0\}\right)^{-1} \widetilde{\mathcal{B}}_{\nu}$ and $f(t)=t^{2 m} g\left(t^{2}\right)^{2}, g(t) \in \mathbb{C}[t]$. Let

$$
P\left(x, \partial_{x}\right)=\frac{1}{v\left(x^{2}\right)} R\left(x, \partial_{x}\right)
$$

for some $R\left(x, \partial_{x}\right) \in \widetilde{\mathcal{B}}_{\nu}$ and $v(x) \in \mathbb{C}[x]$. Set

$$
\operatorname{ord} R\left(x, \partial_{x}\right)=\rho_{1} \quad \text { and } \quad \operatorname{ord}\left(b_{\nu} R\right)\left(x, \partial_{x}\right)=\rho_{2} .
$$

Denote

$$
\begin{aligned}
& \mathcal{S}_{\Psi, 1}=\operatorname{Span}\left\{\frac{1}{v\left(x^{2}\right)} R\left(x, \partial_{x}\right) M\left(x, \partial_{x}\right)(a R)\left(x, \partial_{x}\right) \frac{1}{v\left(x^{2}\right)} \mid M\left(x, \partial_{x}\right) \in \widetilde{\mathcal{B}}_{\nu, \mathrm{sym}}^{l_{1}-\rho_{1}, l_{2}}\right\}, \\
& \mathcal{S}_{\Psi, 2}=\operatorname{Span}\left\{v\left(x^{2}\right) M\left(x, \partial_{x}\right) v\left(x^{2}\right) \mid M\left(x, \partial_{x}\right) \in \widetilde{\mathcal{B}}_{\nu, \mathrm{sym}}^{l_{1}, l_{2}-\rho_{2}}\right\} .
\end{aligned}
$$

Proposition 4.6.

(i) The spaces of differential operators $\mathcal{S}_{\Psi, 1}$ and $\mathcal{S}_{\Psi, 2}$ are subspaces of $\mathcal{B}_{\Psi, \mathrm{sym}}^{l_{1}, l_{2}}$ and are invariant under the transformation $x \mapsto$ $-x$.

(ii) The dimension of the intersection $\mathcal{S}_{\Psi, 1} \cap \mathcal{S}_{\Psi, 2}$ is less than or equal to

$$
\left(l_{1}-\rho_{1}+1\right)\left(l_{2}-\rho_{2}+1\right) \text {. }
$$

Finally we obtain the following theorem. 
THEOREM 4.7. For any self-adjoint (and even, self-adjoint in the case $\nu \in \mathbb{Z}$ ) Darboux transformation from a Bessel function $\Psi_{\nu}(x, z), \Psi(x, z) \in \mathcal{S D}_{\nu}$ the space $\mathcal{S}_{\Psi, 1}+\mathcal{S}_{\Psi, 2}$ consists of differential operators invariant under the transformation $x \mapsto-x$

$$
\operatorname{dim}\left(\mathcal{S}_{\Psi, 1}+\mathcal{S}_{\Psi, 2}\right) \geq\left(l_{1}+1\right)\left(l_{2}+1\right)-\rho_{1} \rho_{2} .
$$

In particular the dimension of the subspace of $\mathcal{B}_{\Psi, \mathrm{sym}}^{l_{1}, l_{2}}$ of differential operators invariant under $x \mapsto-x$ is greater than or equal to $\left(l_{1}+1\right)\left(l_{2}+1\right)-\rho_{1} \rho_{2}$.

Theorems 4.7 and 2.3 imply our final result for Darboux transformations from the Bessel functions:

THEOREM 4.8. Let $\Psi(x, z) \in \mathcal{S D}_{\nu}$ be a self-adjoint (and in addition even if $\nu \in \mathbb{Z})$ Darboux transformation from the Bessel function $\Psi_{\nu}(x, z)$, given by $(4.10)$, (4.11), (4.12), (4.17). Let $\Gamma_{1}, \Gamma_{2}$ be two connected finite contours that do not contain the roots of the polynomials $v(t)$ and $g(t)$, respectively and such that $e\left(\Gamma_{i}\right)=-e\left(\Gamma_{i}\right)$.

Then the integral operator with kernel

$$
K(x, y)=\int_{\Gamma_{2}} \Psi(x, z) \Psi(y, z) \mathrm{d} z
$$

on $L^{2}\left(\Gamma_{1}\right)$ commutes with a formally symmetric differential operator with rational coefficients of order less than or equal to $2\left(\rho_{1} \rho_{2}+1\right)$ and domain all smooth functions on $\Gamma_{1}$.

\section{References}

1. M. Adler, T. Shiota, and P. van Moerbeke, Random matrices, Virasoro algebra, and noncommutative KP, Duke Math. J. 94 (1998), no. 2, 379-431.

2. M. Adler and P. van Moerbeke, The spectrum of coupled random matrices, Ann. Math. 149 (1999), no. 3, 931-976.

3. B. Bakalov, E Horozov, and M. Yakimov, General methods for constructing bispectral operators, Phys. Lett A. 222 (1996), no. 1-2, 59-66.

4. 190 (1997), no. 2, 331-373.

5. Highest weight modules over the $W_{1+\infty}$ algebra and the bispectral problem, Duke Math. J. 93 (1998), no. 1, 41-72.

6. A. Borodin and P. Deift, Fredholm determinants, Jimbo-Miwa-Ueno $\tau$-functions, and representation theory, Comm. Pure Appl. Math. 55 (2002), no. 9, 1160-1230.

7. J. J. Duistermaat and F. A. Grünbaum, Differential equations in the spectral parameter, Comm. Math. Phys. 103 (1986), no. 2, 177-240.

8. E. Frenkel, V. Kac, A. Radul, and W. Wang, $W_{1+\infty}$ and $W\left(\mathrm{gl}_{N}\right)$ algebra with central charge $N$, Comm. Math. Phys. 170 (1995), no. 2, 337-357.

9. F. A. Grünbaum, Time-band limiting and the bispectral problem, Comm. Pure Appl. Math. 47 (1994), no. 3, 307-328.

10. — Band-time-band limiting integral operators and commuting differential operators, Algebra i Analiz 8 (1996), no. 1, 122-126 (Russian); English transl., St. Petersburg Math. J. 8 (1997), no. 1, 93-96.

11. __ Some bispectral musing, The Bispectral Problem (Montréal, 1997), CRM Proc. Lecture Notes, vol. 14, Amer. Math. Soc., Providence, RI, 1998, pp. 31-45.

12. J. Harnad and A. R. Its, Integrable Fredholm operators and dual isomonodromic deformations, Comm. Math. Phys. 226 (2002), no. 3, 497-530.

13. J. Harnad, C. A. Tracy, and H. Widom, Hamiltonian structure of equations appearing in random matrices, Low-Dimensional Topology and Quantum Field Theory (Cambridge, 1992), NATO Adv. Sci. Inst. Ser. B Phys., vol. 315, Plenum, New York, 1993, pp. 231-245.

14. A. Kasman and M. Rothstein, Bispectral Darboux transformations: the generalized Airy case, Phys. D 102 (1997), no. 3-4, 159-176. 
15. H. J. Landau and H. O. Pollak, Prolate spheroidal wave functions, Fourier analysis and uncertainty. II, Bell System Tech. J. 40, (1961), 65-84.

16. _ Prolate spheroidal wave functions, Fourier analysis and uncertainty. III, Bell System Tech. J. 41, (1961), 1295-1336.

17. J. C. McConnell and J. C. Robson, Noncommutative Noetherian rings, Pure Appl. Math., Wiley, Chichester, 1987.

18. J. Palmer, Deformation analysis of matrix models, Phys. D 78 (1995), no. 3-4, 166-185.

19. D. Slepian and H. O. Pollak, Prolate spheroidal wave functions, Fourier analysis and uncertainty. I, Bell System Tech. J. 40, (1961), 43-63.

20. C. A. Tracy and H. Widom, Level spacing distributions and the Airy kernel, Comm. Math. Phys. 159 (1994), no. 1, 151-174.

21. Level spacing distributions and the Airy kernel, Comm. Math. Phys. 161 (1994), no. 2, 289-309.

22. $\ldots$ Fredholm determinants, differential equations and matrix models, Comm. Math. Phys. 163 (1994), no. 1, 33-72.

23. G. Wilson, Bispectral algebras of commuting ordinary differential operators, J. Reine Angew. Math. 442 (1993), 177-204.

Department of Mathematics, University of California at Berkeley, Berkeley, CA 94720, USA

E-mail address: grunbaum@math.berkeley.edu

Department of Mathematics, University of California at Santa Barbara, Santa Barbara, CA 93106, USA

E-mail address: yakimov@math.ucsb.edu 